\title{
Team per l'allestimento degli accessi vascolari: la nostra esperienza
}

\author{
Fabio Borin ${ }^{1}$, Marina Cornacchiari², Barbara Gidaro ${ }^{2}$, Antonia Stasi ${ }^{2}$, Daniele Dal Ry ${ }^{1}$, Anna Mudoni ${ }^{3}$, \\ Maria Giuseppina Ponticelli' ${ }^{2}$, Carlo Guastoni ${ }^{2}$ \\ ${ }^{1}$ Azienda Ospedaliera Legnano, reparto di Chirurgia Vascolare, Legnano (MI) \\ ${ }^{2}$ Azienda Ospedaliera Legnano, reparto di Nefrologia e Dialisi, Legnano (MI) \\ ${ }^{3}$ Reparto di Nefrologia e Dialisi, Ospedale Cardinale G. Panico, Tricase (LE)
}

\section{Team for vascular access: our experience}

In recent years, in the Dialysis departments, the need of a team for the construction and the surveillance of vascular access begins to appear. In this article we describe our experience.

Keywords: Hemodialysis, Team, Vascular access

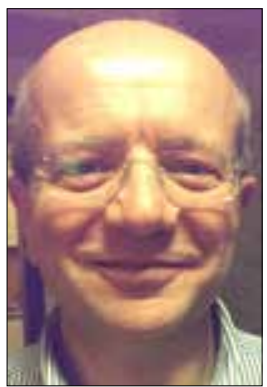

Fabio Borin

\section{Introduzione}

L'incidenza e la prevalenza aumentate (nel mondo) della popolazione emodializzata hanno determinato una crescente richiesta di un accesso vascolare efficiente, di lunga durata e con un minor numero di complicanze. Purtroppo, spesso, condizioni anatomiche vascolari peculiari, comorbilità e l'insorgenza di problematiche che riducono acutamente o progressivamente l'utilizzo di fistole artero-venose allestite con vasi nativi richiedono un ulteriore impegno da parte del nefrologo al fine di assicurare un'adeguata efficienza dialitica. Pertanto, in questi ultimi anni, si va delineando sempre di più la figura di un nefrologo con il ruolo di coordinatore del team dell'accesso vascolare, nel tentativo di migliorare la qualità di vita dell'emodializzato. Infatti, la nostra esperienza ci può far affermare che proprio del team nefrologico è la responsabilità ultima di tutte le complicanze che causano gli svariati ricoveri ospedalieri dei pazienti in emodialisi, con un forte impatto sulla morbilità e sulla mortalità, è propria del

\section{Accepted: May 15, 2015}

Published online: January 21, 2016

Indirizzo per la corrispondenza:

Marina Cornacchiari

Servizio di Nefrologia e Dialisi

Ospedale G. Fornaroli

Via Donatore di Sangue 51

20013 Magenta (MI)

marina.cornacchiari@ao-legnano.it team nefrologico (1) e che solo una stretta collaborazione tra $\mathrm{i}$ vari professionisti dedicati a questo importante device dialitico (chirurgo vascolare, nefrologo e radiologo "interventista") può permettere di trovare soluzioni di fronte a pazienti in cui una fistola tradizionale risulti di impossibile o difficile allestimento, personalizzando l'accesso vascolare e rendendolo, nel contempo, adeguato alla dialisi (2-10). Ciò permette, in molti casi, di prolungare la sopravvivenza della fistola artero-venosa attraverso l'individuazione di percorsi alternativi condivisi, come nei casi clinici riportati qui di seguito.

Nella nostra azienda ospedaliera afferiscono oltre 200 emodializzati ed esiste da anni un'integrazione tra il reparto di nefrologia e quello di chirurgia vascolare, per la pianificazione e le possibili strategie chirurgiche on time riguardanti l'allestimento degli accessi vascolari di maggiore difficoltà. Un importante ruolo svolge la conoscenza della metodica ecocolordoppler, che aiuta a delineare la complessa anatomia dell'accesso vascolare $(8,9)$.

Riportiamo la nostra esperienza con la descrizione di alcuni casi clinici che riteniamo istruttivi.

\section{Casi clinici}

\section{$1^{\circ}$ caso clinico}

Uomo di anni 52 con anamnesi positiva per ipertensione arteriosa, cardiopatia ischemica trattata con $\mathrm{PCl}$ e stenting medicato su IVA e IRC secondaria a LES. Inizia trattamento emodialitico a 46 anni utilizzando come accesso vascolare una FAV distale L-T sinistra. In un momento non precisato della vita di tale accesso vascolare, si sviluppa, in modo lento, una trombosi della vena cefalica dell'avambraccio, a circa 10 $\mathrm{cm}$ dall'anastomosi artero-venosa. Obiettivamente, la mano 
del paziente non presenta una sindrome da iperafflusso evidente, è lievemente più calda e non è edematosa né dolente, rispetto alla controlaterale.

Inoltre, all'esame obiettivo, il tratto di vena cefalica distale alla trombosi si presenta dilatato, ma non aneurismatico. Tale tratto del vaso viene, da tempo, utilizzato per il posizionamento dell'ago arterioso con ottimi flussi in aspirazione. Anche la vena dorsale della mano è dilatata ma non aneurismatica. L'ago venoso è da tempo inserito nella vena basilica a livello della piega del gomito.

Lo studio ECD documenta come la trombosi sia estesa dal terzo medio della vena cefalica fino alla piega del gomito e che il flusso è deviato verso la vena dorsale della mano e verso alcuni circoli collaterali che da essa prendono origine. Questi vasi sono arterializzati e permettono al flusso di scaricare nella vena basilica del braccio. Si decide di mantenere un atteggiamento di attesa. A distanza di alcuni mesi, comincia a comparire una sindrome da iperafflusso con edema ingravescente e dolore alla mano e si rende, quindi, necessario un intervento terapeutico.

L'interposizione di una protesi retta, a ponte tra vena cefalica distale e prossimale, a superare il tratto occluso, può essere la scelta più immediata. La pervietà dell'anastomosi e del tratto più distale della vena cefalica, associata alla presenza di una vena dorsale della mano arterializzata e di calibro congruo fa prospettare un'altra soluzione, di maggiore difficoltà tecnica, ma in grado di evitare l'uso di materiale protesico. Tale possibilità consiste nella sezione e nella trasposizione del ramo dorsale della cefalica, sufficientemente lungo, che è innestato per sostituire il tratto trombizzato della cefalica, a ponte, quindi, con la mediana basilica (Fig. 1).

La FAV, a distanza di 5 anni, è ancora funzionante ed è scomparsa la sindrome da iperafflusso.

\section{$2^{\circ}$ caso clinico}

Donna di anni 38. Nell'anamnesi, da segnalare: epatite $B$ nell'infanzia e pielonefriti recidivanti con comparsa di IRC progressiva. A 19 anni, inizio del trattamento emodialitico effettuato tramite un accesso vascolare nativo confezionato all'arto superiore sinistro in un altro centro. Pochi mesi dopo l'inizio della dialisi, trapianto renale da donatore cadavere. Giunge alla nostra attenzione nel Settembre del 2010, in seguito a una trombosi venosa profonda dell'asse iliacofemoro-popliteo destro e contestuale necessità di riavviare, in urgenza, con l'utilizzo di un catetere venoso centrale temporaneo, un trattamento emodialitico per peggioramento funzionale (IRC stadio 5 secondaria a esaurimento funzionale del rene trapiantato). Si programma, successivamente, un esame ECD per allestire un accesso vascolare nativo. L'ECD mostra, a livello dell'arto superiore sinistro, un circolo venoso superficiale non utilizzabile per la presenza di vasi particolarmente filiformi e fibrotici. A destra, la vena cefalica dell'avambraccio presenta un buon calibro $(3.5 \mathrm{~mm}) \mathrm{ma}$

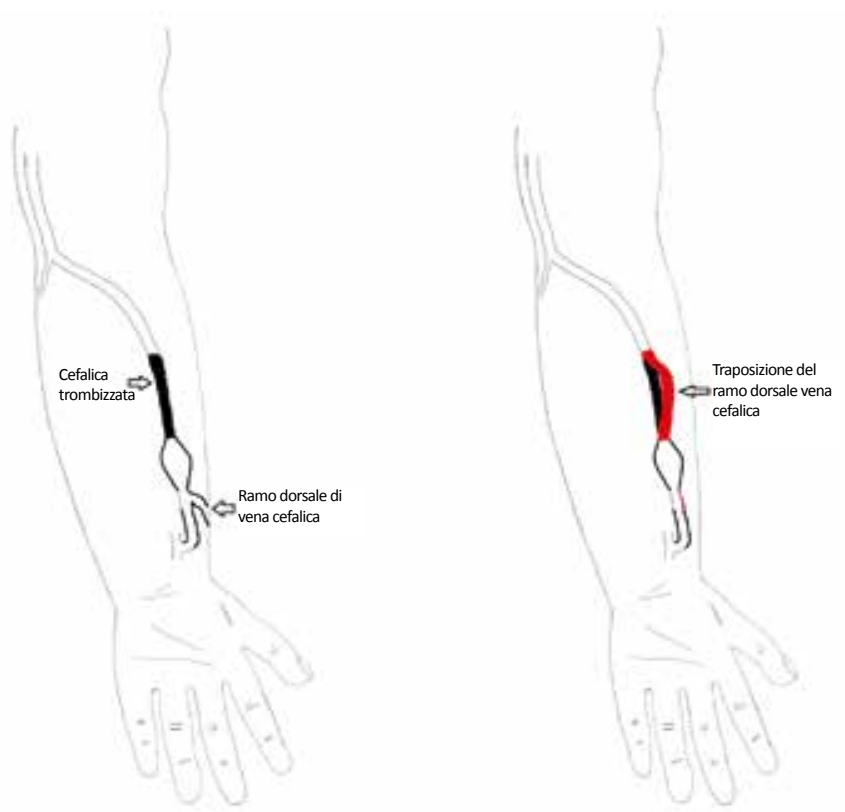

Fig. 1 - Trasposizione del ramo dorsale della vena cefalica.

è subocclusa a partire dai due terzi superiori del braccio. La vena basilica è esile nell'avambraccio, ma di calibro superiore ai $4 \mathrm{~mm}$ a livello del braccio. Non si evidenziano né la mediana cefalica né la mediana basilica e, pertanto, non è presente alcuna comunicazione tra la vena cefalica e la basilica a livello della piega del gomito. Un accesso protesico potrebbe essere interposto tra arteria omerale destra e vena basilica, ma abbiamo deciso di riservare tale soluzione come futura opzione, considerata la giovane età della paziente e la probabile necessità di accessi vascolari. Pertanto, si confeziona una FAV distale L-T tra arteria radiale e cefalica dell'avambraccio. A livello prossimale, si isola la cefalica del braccio nel terzo inferiore, dove ha un calibro di $3.5 \mathrm{~mm}$, la si mobilizza e la si traspone medialmente anastomizzandola alla vena basilica (Fig. 2). L'accesso vascolare è rimasto ben funzionante per 3 anni. In seguito a un episodio ipotensivo prolungato, si è, poi, trombizzato. È stato, successivamente, sostituito da un accesso vascolare protesico tra arteria omerale sinistra e vena basilica.

\section{$3^{\circ}$ caso clinico}

Donna di anni 74. Nell'anamnesi si segnalano: diabete mellito di tipo II con neuropatia periferica, micro-macroangiopatia e nefropatia, cirrosi epatica HCV-correlata con pregressa resezione sesto segmento epatico per epatocarcinoma, ipertensione arteriosa, BPCO e cardiopatia ipertensiva. È iniziato un trattamento emodialitico sostitutivo tramite un catetere venoso centrale e viene programmato uno studio ECD. L'esame mostra, a destra, un circolo venoso superficiale con vasi esigui e fibrotici, non utilizzabili per 


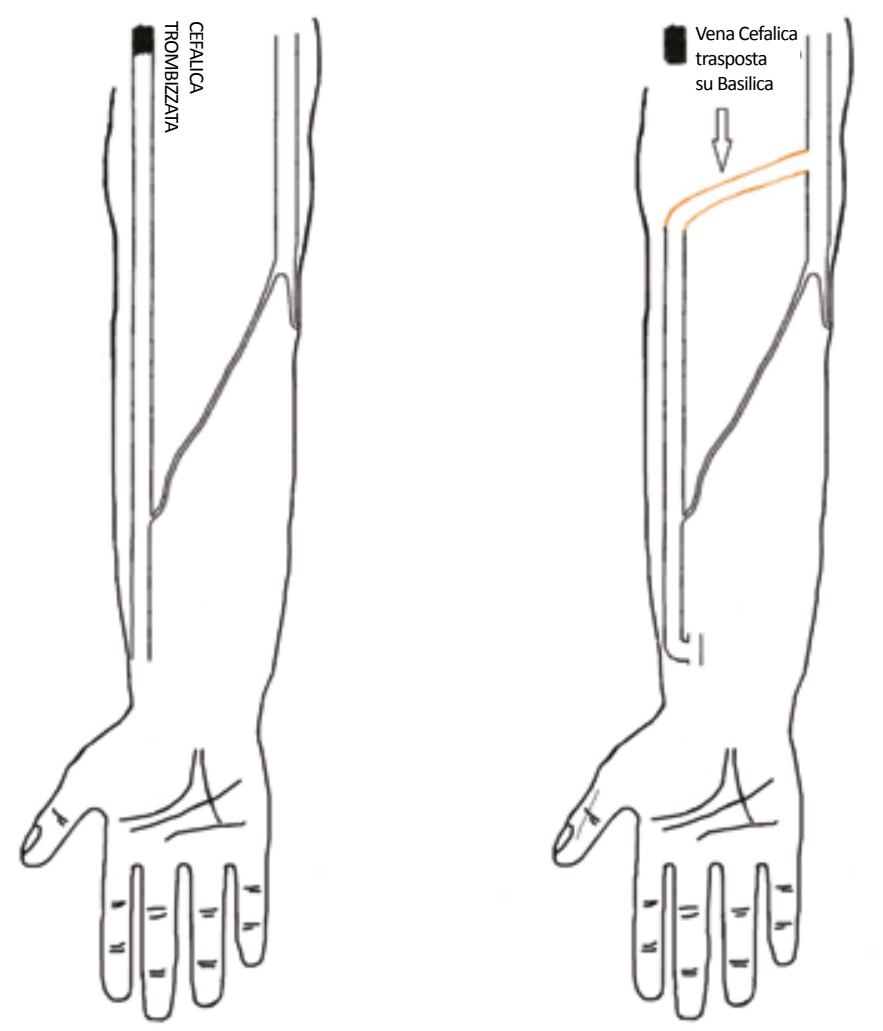

Fig. 2 - Vena cefalica del braccio mobilizzata e trasposta su vena basilica.

la costruzione di una FAV distale e/o prossimale sia nativa che protesica. Unica possibilità a destra potrebbe essere il possibile posizionamento di un tratto protesico tra omerale e ascellare. A sinistra, la vena cefalica dell'avambraccio è fibrotica al terzo inferiore e di buon calibro al terzo medioprossimale ed è occlusa alla piega del gomito. La vena basilica sinistra è di calibro esile nell'avambraccio e al terzo distale del braccio, successivamente presenta un calibro di $4 \mathrm{~mm}$ fino al suo sbocco in vena ascellare. Unica possibilità a sinistra è sempre un accesso vascolare protesico a ponte tra omerale e basilica al terzo medio e/o prossimale. Si ritiene, invece, di poter fare un tentativo di trasporre la vena grande safena della gamba destra a ponte tra la vena cefalica al gomito immediatamente prima della sua occlusione e la vena basilica del braccio al terzo medio. Un'anastomosi artero-venosa latero-terminale è confezionata al terzo medio dell'avambraccio tra arteria radiale e vena cefalica. Abbiamo scelto di utilizzare la vena grande safena del lato destro preservando quella sinistra di miglior calibro per eventuali bypass cardiaci, valutate le comorbilità della nostra paziente $\mathrm{e}$, in generale, per la frequenza di problematiche cardiologiche nei nostri pazienti emodializzati (Fig. 3). La FAV ha avuto una sopravvivenza limitata di soli 8 mesi per trombosi della vena basilica con comparsa di sindrome da iperafflusso dell'avambraccio e della mano. È stata chiusa ed è stato riposizionato un catetere venoso centrale.

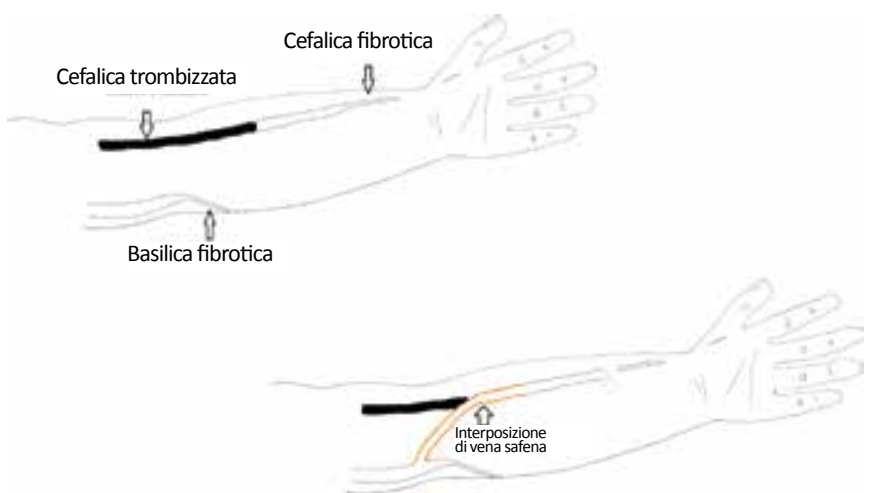

Fig. 3 - Vena safena messa a ponte tra vena cefalica dell'avambraccio e vena basilica del braccio.
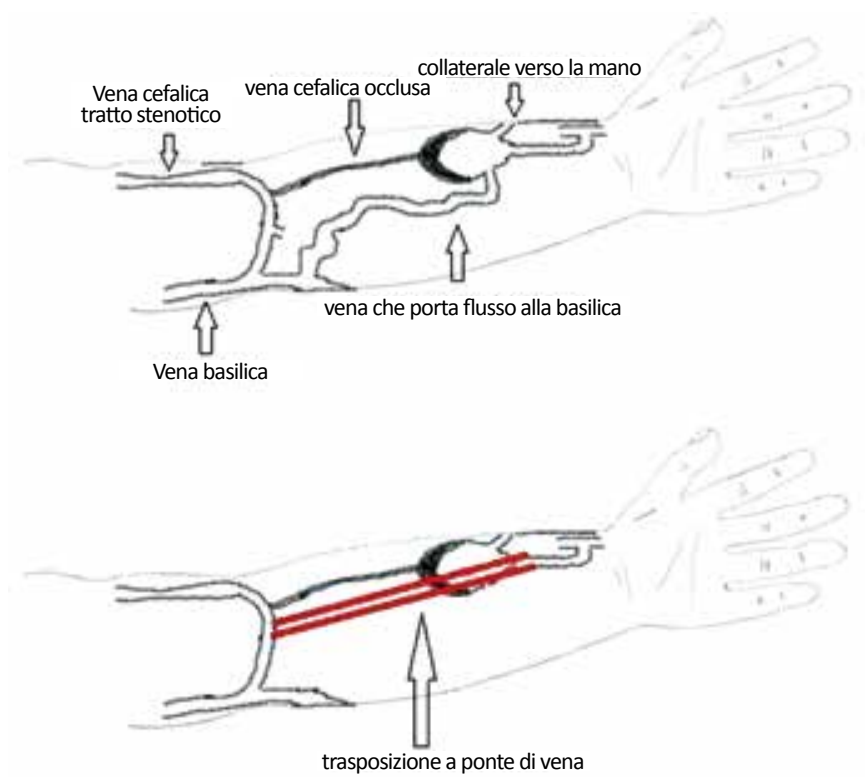

Fig. 4 - Vena collaterale mobilizzata e posta a ponte tra vena cefalica al terzo inferiore e vena mediana basilica.

\section{$4^{\circ}$ caso clinico}

Uomo di anni 50. Pregresso trapianto di fegato per cirrosi etilica e HCV-correlata. Diabete mellito in terapia insulinica, ipertensione arteriosa, IRC terminale da nefropatia diabetica, polineuropatia diabetica. In dialisi dall'età di 44 anni tramite una FAV distale sinistra L-T radiocefalica. II paziente dializza in un CAL. Dopo circa 3 anni di buon funzionamento della FAV, compaiono una flebite a livello di una piccola dilatazione aneurismatica localizzata a circa $10 \mathrm{~cm}$ dall'anastomosi artero-venosa e un edema ingravescente della mano. $L^{\prime} E C D$ mostra pervietà dell'anastomosi e parziale trombosi dell'aneurisma, oltre al quale la vena cefalica è occlusa fino alla piega del gomito, verosimilmente da diverso tempo (Fig. 4). A questo livello, sia la vena mediana cefalica che la mediana basilica sono pervie e proseguono rispettivamente nel- 
la cefalica e nella basilica del braccio. La vena cefalica del braccio non presenta un flusso arterializzato e al terzo medio è stenotica. Dall'aneurisma si dipartono dei vasi collaterali: uno si dirige alla mano e si rende responsabile della presenza di un modesto edema e un altro, ben sviluppato e a decorso tortuoso, decorre sulla faccia antero-mediale dell'avambraccio e sbocca nella vena basilica dell'avambraccio al terzo superiore, alimentando, in questo modo, la basilica stessa che presenta un flusso arterializzato. II paziente era venopunto da tempo, con l'ago arterioso posizionato nel tratto distale della cefalica a livello dell'aneurisma e con l'ago venoso nella basilica a livello della piega del gomito.

Abbiamo, così, analizzato le possibilità chirurgiche in questo paziente: 1) posizionamento di una protesi tra vena cefalica distale, nel tratto che precede l'aneurisma e la vena basilica e 2) allestimento di una FAV prossimale con la perforante con flusso che verosimilmente si sarebbe diretto in prevalenza verso la basilica, a causa della stenosi sul decorso della cefalica.

Sempre considerando la giovane età del paziente e la mancanza di prospettive di trapianto renale, abbiamo preferito tentare, anche in questo caso, una soluzione tecnicamente difficoltosa. Abbiamo quindi isolato la vena arterializzata che si era sviluppata a partire dall'aneurisma e l'abbiamo trasposta a ponte, sostituendo così il tratto di vena cefalica trombizzata (Fig. 4). La FAV è ben funzionante da 9 mesi.

\section{$5^{\circ}$ caso clinico}

Uomo di anni 46. IRC secondaria a malattia policistica renale, ipertensione arteriosa. L'ECD mostra bilateralmente una vena cefalica inesistente sia a livello del braccio che a livello dell'avambraccio; a sinistra, la vena basilica dell'avambraccio presenta un calibro nell'avambraccio di $2.2 \mathrm{~mm}$, mentre la destra è esile. Viene allestita, quindi, una fistola artero-venosa latero-terminale sin, tra arteria ulnare e vena basilica. A distanza di due anni, la FAV è ancora funzionante. L'alternativa all'accesso realizzato sarebbe stato l'allestimento di una fistola prossimale tra omerale e basilica con superficializzazione del vaso o l'impianto di una protesi tra omerale e basilica, ma, in considerazione della giovane età, è stata lasciata per tempi futuri.

\section{Discussione}

I primi quattro casi succitati, allestiti tra l'agosto del 2010 e il marzo del 2015 , si riferiscono a trasposizioni e/o ad autotrapianti venosi. Si tratta di casi in cui l'opzione terapeutica più immediata è il posizionamento di una protesi, ma una protesi necessita di una maggiore attenzione e manutenzione sia in termini di venopuntura che di controlli nel tempo e ha una minore sopravvivenza rispetto alla fistola nativa. Abbiamo, quindi, preferito riservare l'accesso protesico per tempi successivi.

$\mathrm{Nel} 1^{\circ}$ e nel $4^{\circ}$ caso in particolare, abbiamo ripristinato la funzionalità dell'accesso vascolare, risolvendo la sindrome da iperafflusso presente, utilizzando una vena che nel frattempo si era arterializzata.

Certamente, tale intervento chirurgico è stato più indaginoso, con un maggiore rischio per problemi di sanguinamento o di trombosi a causa di una possibile torsione della vena trasposta. D'altro canto, se ben riuscito, l'intervento permette di recuperare una vena che, diversamente, sarebbe andata persa.

Si evince, dalla descrizione dei casi, che, in due pazienti, una più attenta sorveglianza dell'accesso vascolare, attraverso un più accurato esame obiettivo supportato dalla valutazione ECD, avrebbe dovuto precocemente mettere in evidenza la stenosi sul decorso della cefalica arterializzata, vaso che, con il tempo, si è ostruito completamente. Da qui, la riflessione che una diagnosi più precoce avrebbe permesso di intervenire con una PTA della vena, metodica non più indicata successivamente nel momento in cui l'occlusione si è trasformata in completa e inveterata. II buon funzionamento dell'accesso vascolare, nonostante la problematica, e l'assenza per lungo tempo di una sintomatologia evidente sono concause che ne hanno posticipato la diagnosi.

Con la descrizione di questi casi clinici, vogliamo sottolineare l'utilità, a nostro avviso, di fronte all'allestimento o al riallestimento di una FAV, di individuare diverse soluzioni alternative, scegliendo quella che possa tentare e permettere una migliore sopravvivenza nel tempo dell'accesso vascolare o una migliore venopuntura, soprattutto in quei pazienti che non possono essere avviati al trapianto renale e che, di conseguenza, possono aver bisogno, negli anni a venire, di nuovi accessi vascolari.

La presenza di un team degli accessi vascolari riveste, come da più parti viene riconosciuto, un ruolo importante in tal senso e l'integrazione consente il raggiungimento di risultati migliori. Questo team dovrebbe comprendere figure con competenze variamente suddivise, in ecocolordoppler, chirurgia vascolare e radiologia interventistica. Un team siffatto, sebbene auspicabile, non è, però, sempre presente.

Nella nostra esperienza, il team è composto, attualmente, da 2 figure che lavorano nella stessa azienda ospedaliera, il nefrologo interventista e il chirurgo vascolare, entrambi esperti in ecografia vascolare, e si occupano in prima persona dell'allestimento degli accessi vascolari.

Nel nostro team, la persona designata a proporre la tipologia dell'accesso vascolare è il nefrologo che esegue I'ECD per lo studio dei vasi e che si occupa anche dell'allestimento delle fistole routinarie.

È sempre il nefrologo che, conoscendo a fondo la storia clinica, le caratteristiche del paziente e anche tutte le problematiche dell'accesso vascolare, propone soluzioni alternative per il singolo paziente sia nel momento del primo confezionamento di un accesso vascolare sia quando quest'ultimo presenta delle problematiche. Di fronte ad accessi vascolari protesici o particolari come sono le trasposizioni di vasi succitate, tecnicamente difficoltose, il nefrologo si interfaccia con il chirurgo vascolare per studiare a tavolino la strategia migliore. È il chirurgo vascolare che, per la maggiore esperienza, mette in evidenza eventuali 
problematiche che possono svilupparsi ed eventualmente "sceglie la proposta migliore anche da un punto di vista tecnico".

Questo lavoro di equipe si realizza perché, tra i due colleghi, si è instaurato un rapporto basato sulla fiducia e sulla stima reciproca delle proprie capacità e competenze, che permette di discutere i diversi casi clinici e condividere le proposte, migliorarle e modificarle, individuando eventuali punti di criticità con l'impegno per la risoluzione.

Attraverso la discussione dei casi e l'integrazione delle singole specialità, è stato possibile, negli anni, recuperare accessi vascolari che avrebbero potuto essere considerati persi e creare soluzioni alternative, in presenza di situazioni particolari, come nei casi clinici riportati.

Alla figura del chirurgo vascolare e del nefrologo "interventista" andrebbe aggiunta quella del radiologo interventista, che, nella nostra realtà, è stato per molti anni un collega dislocato in un altro ospedale con cui la collaborazione è sempre stata ottima, Da circa un anno, è, però, in atto la formazione presso il nostro nosocomio di questa terza figura del team.

\section{Conclusioni}

La stretta collaborazione fra i componenti del team dedicato all'accesso vascolare rende possibile, grazie a un'adeguata comunicazione tra i professionisti, la realizzazione di un accesso vascolare non solo funzionante, ma personalizzato e adeguato alle esigenze dialitiche, ma anche l'esecuzione di un numero sempre crescente di procedure che permette di migliorare le competenze, soprattutto in termini di efficacia e sicurezza.

Inoltre, riteniamo che un accurato studio ECD in fase preoperatoria, attraverso la conoscenza dell'anatomia vascolare, permette la pianificazione di soluzioni alternative in grado di evitare il ricorso al materiale protesico $\mathrm{o}$ al posizionamento di cateteri a permanenza sia nel momento dell'allestimento ex-novo di una FAV che in presenza di complicanze dell'accesso stesso $(8,9)$.

\section{Disclosures}

Financial support: No financial support was received for this submission. Conflict of interest: The authors have no conflict of interest.

\section{Bibliografia}

1. Lomonte $\mathrm{C}$, Basile $\mathrm{C}$. The role of the nephrologist in the management of vascular access. Nephrol Dial Transplant 2011; 26:1461-3.

2. Spergel LM, Ravani P, Asif A, Roy-Chaudhury P, Besarab A. Autogenous arteriovenous fistula options. J Nephrol 2007;20: 288-98.

3. Spergel LM, Ravani P, Roy-Chaudhury P, Asif A, Besarab A. Surgical salvage of the autogenous arteriovenous fistula (AVF). J Nephrol 2007;20:388-98.

4. Dorobantu LF, Iliescu VA, Stiru O, Bubenek S, Novelli E. The brachio-brachial arteriovenous fistula: mid-term results. J Vasc Access 2010;11(1):23-5.

5. Cavatorta F, Galli S, Zollo A, Crespi E, Carnabuci A. Ulno-basilic arteriovenous fistulae: indications and surgical technique. J Vasc Access 2008;9:73-80.

6. Bosticardo GM, Schillaci E, Berto IM, Cravero R, Gheller $G$, Bercia R. Forearm vein transposition for hemodialysis autogenous vascular access. J Vasc Access 2011;12(1):73-5.

7. Nguyen VD, Lawson L, Ledeen M, et al. Successful multidisciplinary interventions for arterio-venous fistula creation by Pacific Northwest renal Network 16 vascular access quality improvement program. J Vasc Access 2007;8:3-11.

8. Davidson I, Chan D, Dolmatch B, et al. Duplex ultrasound evaluation for dialysis access selection and maintenance: a practical guide. J Vasc Access 2008;9:1-9.

9. Ives $\mathrm{CL}$, Akoh IA, George I, Vaughan-Huxley E, Lawson H. Preoperative vessel mapping and early post-operative surveillance duplex scanning of arteriovenous fistula. J Vasc Access 2009; 10:37-42.

10. Tazza L. Team dell'accesso vascolare: modelli organizzativi. Nepfro Meet, 02 Ottobre 2012. 